\title{
Profile of teriparatide in the management of postmenopausal osteoporosis
}

REVIEW

This article was published in the following Dove Press journal:

International Journal of Women's Health

30 March 2010

Number of times this article has been viewed

\author{
Andrea Sikon' \\ Pelin Batur ${ }^{2}$ \\ 'Cleveland Clinic Lerner COM, \\ Cleveland Clinic, Cleveland, $\mathrm{OH}$, \\ USA ${ }^{2}$ Cleveland Clinic Lerner COM, \\ Cleveland Clinic Independence, \\ Independence, $\mathrm{OH}$, USA
}

\begin{abstract}
One out of every 2 women within postmenopause are at risk of fracture due to osteoporosis. Fortunately, a growing arsenal of therapies is becoming available to treat this disease and prevent fracture. A new class of anabolic agents has emerged within the last decade that brought with it a new concept in osteoporosis therapy: building new stronger bone rather than simply inhibiting bone turnover. Evidence is accumulating to understand how to best utilize these new agents, and which patients benefit most. This article will review the effectiveness, risks, timing and clinical uses of teriparatide in postmenopausal osteoporosis.
\end{abstract}

Keywords: osteoporosis, menopause, teriparatide

\section{Background}

With the aging population and the resultant expected rise in fracture prevalence in the near future, there is growing interest in expanding therapies for osteoporosis (OP). Although OP occurs in men and younger women, postmenopausal women remain most effected, with a lifetime risk of fracture of $50 \%{ }^{1}$ The past 2 decades have seen incredible innovation in the bone field. The 1990s brought the bisphosphonates (BPS), a class of bone specific agents with versatile dosing options, impressive efficacy, and relative safety. Other advances in OP treatment came when estrogen was proven to prevent hip fracture in the Women's Health Initiative, but enthusiasm was limited by the discovery of potential adverse effects likely associated with timing of initiation that limits its more widespread use. The selective estrogen receptor modulator class was discovered and expanded, but has limitations due to side effects, gender restrictions, and the lack of hip fracture reduction. Although multiple drugs are available to treat OP, they had all previously belonged to the anti-catabolic group, acting by inhibiting bone turnover. An entirely new class of therapeutic agents for OP was announced in 2002 with the Food and Drug Administration (FDA) approval of teriparatide, the first medication available with an anabolic mechanism.

Teriparatide (Forteo ${ }^{\circledR}$ [US], Forsteo ${ }^{\circledR}$ [Europe]; Eli Lilly and Co.) is a recombinant Director of Primary Care Women's Health-Medicine Institute, ChairDepartment of Internal Medicine, Certified Clinical Densitometrist, Assistant Professor of Medicine, Cleveland Clinic Lerner COM, Cleveland Clinic, 9500 Euclid Ave. G I0, Cleveland, $\mathrm{OH} 44195$, USA

Tel +l 216-444-9896

Fax + I 216-434-2334

Email sikona@ccf.org formulation of endogenous parathyroid hormone (PTH), containing a 34-amino-acid sequence which is identical to the N-terminal portion of the human hormone [rhPTH(1-34)]. Though human recombinant parathyroid hormone (rhPTH [1-84]) (Preotact ${ }^{\circledR} ;$ Nycomed) and synthetic human parathyroid hormone (hPTH [1-34]) are other anabolic agents that have also been used in clinical trials, they are not approved for use in the United States (US) at this time, and so we will focus on teriparatide in our review. 


\section{Efficacy}

This fragment known as teriparatide (1-34), retains the bone-forming effects of the full-length human PTH, stimulating osteoblast function, increasing gastrointestinal calcium absorption, and increasing renal tubular reabsorption of calcium. When considering the significant bone loss that can occur with the disease hyperparathyroidism, the treatment of OP with the use of a PTH analogue may initially seem counterintuitive. Indeed, continuous high levels of PTH do mobilize calcium from the bone and accelerates bone loss; however, pulsatile exposure to lower doses, as occurs with once daily dosing with teriparatide, actually results in increased bone mineral density (BMD) and bone mass, with the improvement seen in both cancellous and cortical bone architecture. ${ }^{2-5}$ Although generalized bone remodeling is stimulated, there is a net favoring of bone formation, possibly due to the inhibition of osteoblast apoptosis or the transformation of bone lining cells back to functional osteoblasts. ${ }^{6,7}$ This is reflected in bone marker behavior with teriparatide therapy. Bone formation markers rise more rapidly and peak within the first 3 months of therapy with teriparatide, as compared to the bone resorption markers, which peak later at 6 months. This delay between bone formation overriding bone resorption is referred to as the "anabolic window." This is an important concept to understand as it has significant potential implications on optimal dosing duration and sequencing. This initial favoring of bone formation results in new bone that has increased strength and microarchitecture. Skeletal areas comprised of cancellous bone (lumbar spine) see more rapid and quantitative increases overall from teriparatide therapy than sites of cortical bone (femoral neck and radius). ${ }^{8}$

Teriparatide is FDA approved for the treatment of OP in postmenopausal women at highest risk for fracture, primary or hypogonadal OP in men at high fracture risk, and glucocorticoid-induced osteoporosis (GIOP) in both men and women. As with most studies of therapies for OP, proving true fracture reduction, which in the end is the most important outcome, is inherently difficult. Such studies require large sample sizes over many years, which are difficult to complete. Thus, surrogate markers such as BMD and bone formation and turnover markers are often used to assess pharmacologic efficacy.

Teriparatide at $20 \mu \mathrm{g} /$ day has been proven to decrease spine and nonvertebral fractures except hip fractures in postmenopausal women with prior vertebral fractures, reducing the incidence of new vertebral fractures as identified by visual semiquantitative readings by $65 \%$, and nonvertebral fractures by $53 \%$ compared to placebo, after 12 months of therapy. Dosing with $40 \mu \mathrm{g} / \mathrm{day}$ was not superior and thus this dose was not approved. ${ }^{9}$ When quantitative morphometry was also used to diagnose the fractures, an even higher vertebral fracture reduction of $84 \%$ was seen. ${ }^{10}$

Multiple scenarios are raised when considering therapy with teriparatide: use following other prior therapies, use in those who are on concomitant therapy with other agents, and, least commonly, use in the treatment-naïve group. Reasons to consider switching therapy to teriparatide from other therapies may be new fracture or loss of BMD on current therapy and/or drug intolerance. Of course, a true failure of therapy must first include a re-evaluation of potentially new secondary causes and compliance with not only the bone agent but also with calcium and vitamin D supplementation.

\section{Sequential therapy}

Sequential therapy is often considered in patients who are already on an anti-resorptive medication but have subsequently fractured or continued to lose bone mass leading to the decision to switch to an anabolic agent. Another scenario are those who have an intolerance or a contraindication to one of the other bone agents, although less common now with the use of intravenous (IV) zoledronic acid (Reclast ${ }^{\mathbb{B}}$; Novartis) and IV ibandronate (Boniva ${ }^{\circledR}$; Genentech), which can circumvent gastrointestinal contraindications to BPS, as well as compliance issues. Some even consider switching to an anabolic agent in severe OP if there remains increased bone turnover by marker assessment, despite treatment with an IV BPS that does not rely on oral absorption.

Teriparatide appears to retain its anabolic effect in postmenopausal women with OP previously treated with long term BPS, although it is believed that the benefits is probably less than that seen in previously untreated patients, with less relative increases in BMD and markers of bone formation. ${ }^{11}$ Ettinger et al studied postmenopausal women who had previously received alendronate or raloxifene followed by teriparatide given at $20 \mu \mathrm{g} / \mathrm{day}$. Increases in lumbar spine were more robust after 18 months in those previously treated with raloxifene than those treated with alendronate $(10.2 \%$ vs $4.1 \%, P<0.05)$ and increases in mean total hip BMD were seen only in those previously treated with raloxifene. ${ }^{12}$ A study by Eastell compared teriparatide therapy in postmenopausal women with OP who were previously BPS naive, to those who had previously received a BPS, who were further defined as being adequate responders vs inadequate responders to BPS. Inadequate responders were defined as having $>1$ new clinical fragility fracture after 12 months 
of therapy, a T-score $<-3.0$, or a BMD decrease $>3.5 \%$ at either the spine, total hip, or femoral neck after 24 months of therapy. All groups demonstrated significant increases in lumbar spine BMD; however, the greatest response was seen in the treatment naïve group at $13.5 \%$, with $10.8 \%$ in the adequate responders to BPS group and $9.7 \%$ seen in the inadequate responders group. ${ }^{13}$

This blunting of response to PTH therapy following BPS treatment may have to do with the avidity of BPS binding to hydroxyapatite in osteoclasts, which varies by BPS type. This was indeed shown by Miller et al where postmenopausal women with OP were treated with PTH following either alendronate, which has a higher binding affinity, vs risedronate therapy. Significant blunting was seen in the alendronate-treated group, with only $13.7 \%$ increase in quantitative computerized tomography (QCT) of trabecular bone at the spine vs $24 \%$ seen with those who received risedronate, which has known lower binding affinity $(P=0.023) .{ }^{14}$

\section{Treatment-naïve patients}

When comparing teriparatide vs alendronate in postmenopausal osteoporotic women, teriparatide increased BMD at the lumbar spine, femoral neck, and total body to a greater degree than alendronate, but BMD at the one third distal radius decreased. Nonvertebral fracture frequency was significantly lower in the teriparatide treated group (4.1\%) than in the alendronate group $(13.7 \%, P=0.042) .{ }^{15}$ Unfortunately, not many direct comparisons of fracture reduction can be made compared to other antiresorptive agents due to a lack of head-to-head studies looking at fracture outcomes. Saag et al evaluated teriparatide vs alendronate therapy in women and men with GIOP and showed not only greater increases in lumbar spine BMD, but favored teriparatide as being more effective than BPS in preventing vertebral fractures $(1.7 \%$ vs $7.7 \%, P=0.007)$. However, there was no difference in nonvertebral fracture rates. ${ }^{16}$

\section{Combination therapy}

Several studies have evaluated outcomes related to combining teriparatide and other OP therapies in postmenopausal women. Black and colleagues assessed the differences in BMD with rhPTH (1-84) alone, in combination with alendronate, vs alendronate alone in postmenopausal women with low BMD at the hip or spine. This study showed that adding a BPS to an anabolic therapy blunted the response seen in treatment-naïve patients who were given anabolic therapy alone. Although BMD of the spine (measured by DXA and QCT) increased significantly in all treatment groups after 12 months of treatment, the increase in the rhPTH (1-84) only group was approximately twice that found when alendronate was added to rhPTH (1-84) $(25.5 \%$ vs $12.9 \%$; $P=0.01)$. Similarly, bone formation markers increased only in the rhPTH (1-84) group vs. decreased in the combination group. ${ }^{17}$ Similar to findings in postmenopausal women with $\mathrm{OP}$, concomitant therapy with teriparatide and alendronate in men blunted the increase in lumbar spine and femoral neck BMD seen in teriparatide monotherapy. ${ }^{18}$ The study results of Cosman et al challenge the above findings of blunting with concomitant BPS and anabolic therapy. In comparing those who are treated with teriparatide as add on therapy to baseline alendronate or raloxifene use vs. teriparatide alone, this study showed significant decreases in bone turnover with combination therapy, and is still underway via an extension to follow out these changes. ${ }^{19}$

With the advent of FDA approval of less frequent IV BPS options, studies have begun to assess if this anabolic blunting effect differs with variations in BPS dosing intervals. Gasser et al found that when a single high dose of iv zoledronic acid was given followed by daily injections of PTH, there was no blunting of the response to PTH as measured by peripheral QCT. In a separate treatment arm, the same BPS was administered three times per week, and this regimen did cause a blunting of the relative PTH response..$^{20}$ This suggests that intermittent high doses of zoledronic acid given concomitantly with anabolic therapies may not have the same blunting effect as does more regular BPS dosing.

Estrogen and raloxifene do not appear to blunt the anabolic effect of PTH like BPS. In fact, some studies show that combination therapy with these agents has a small additive effect on BMD, with estrogen-hPTH (1-34) treatment resulting in increased vertebral BMD in one study and raloxifenehPTH (1-34) resulting in increased vertebral and total hip BMD in another. ${ }^{21,22}$

In summary, the true impact of combination therapy on fracture rates is unknown. Most combination trials to date have been small trials of short duration with various combinations. Even less is known about the effects of combination therapy in those with GIOP. There are also concerns about potential over-suppression of bone. This is an area of ongoing research. The official stand of the National Osteoporosis Foundation is that the added cost and potential side effects of combination therapy should be weighed against potential gains. ${ }^{1}$

\section{Indications and use}

Teriparatide is indicated in the treatment of OP in postmenopausal women, primary or hypogonadal OP in men, and 
GIOP in both men and women. Specifically, patients should be at high risk of fracture, such as those with a prior fragility fracture, multiple risk factors for fracture, and those who have failed or are intolerant to other meds. Of course, it is important to remember that anyone who fractures while on an antiresorptive medication has not necessarily had a treatment failure since no therapeutic agent has been shown to totally eliminate the risk of fractures. Detailed history and secondary evaluation is always prudent before changing therapy. Teriparatide can also be considered as first line treatment in those who have a T-score $<-3$ if they have other risk factors for fracture, assuming reversible secondary causes have been investigated. ${ }^{23}$ As with other OP therapies, excluding metabolic bone diseases other than primary OP is critical prior to initiating therapy, as this medication is not indicated for metabolic bone disease other than OP at this time. The lack of studies showing hip fracture reduction should not limit the use of teriparatide, given that very few bone agents in clinical use today have been proven to help reduce hip fracture based on prospective randomized studies (with the exception of estrogen therapy: Fosamax ${ }^{\circledR}$; Merck and Co., Inc., and zoledronic acid).

Teriparatide is given as a $20 \mu \mathrm{g}$ subcutaneous injection once daily into the thigh or abdominal wall. Currently, it is not approved for use longer than 2 years in the US (18 months in the EU). The reason for this time limitation is due to the association of teriparatide with an increased risk of osteosarcoma in rat studies. This risk seems to be related to both dose and duration of the medication (see Precautions and contraindications for wider discussion). Patients should be easily able to sit down during initial administration in the event of orthostasis. Transient orthostatic hypotension usually occurs within 4 hours of dosing and it is usually limited to the first several doses. Recommending dosing at bedtime may help to minimize this occurrence. The injection pen was recently redesigned to a simpler design; however, patients should still receive formal training in the proper use of the device prior to initiation. It also requires refrigeration, which can raise storage issues during travel.

Similar to endogenous PTH, teriparatide can cause hypercalcemia; however, this rise is transient due to the pulsatile nature of dosing and short half-life of 1 hour. Rapid peak serum concentration is seen within 30 minutes of administration with good bioavailability (95\%) and subsequent clearance of serum levels within 3 hours. Although unstudied specific to the drug, clearance is presumed to be the same as that of endogenous PTH, with metabolism mainly in the liver with excretion by the kidneys. ${ }^{8,24}$

\section{Cost effectiveness}

Teriparatide is quite expensive compared to most alternative bone agents currently available. At approximately US\$800/month, it can be anywhere from 10 to 30 times more costly than oral BPS therapy, or even greater with the generic formulation of alendronate being available.

As with any medication for OP, which has a variable population prevalence with additional confounders like vitamin D and calcium sufficiency, determining cost effectiveness amongst therapies is complex. Results of cost effectiveness evaluations with teriparatide are likewise based on a number of assumptions, data limitations, and can be difficult to extrapolate to populations beyond those studied. Such is the case for men and those with GIOP, as none yet have evaluated these groups for cost effectiveness.

Cost considerations and questionable effect on hip fracture reduction prohibit using teriparatide as first line therapy for the general population. However, utilizing this medication in women aged $>65$ with $\mathrm{BMD}<-3$ and recent fragility fractures has been shown to be an effective economic option when considering quality adjusted life-years vs no therapy. ${ }^{23}$

\section{Precautions and contraindications}

Data are limited for the use of this medication in those with cardiovascular, hepatic, or liver disease; thus, caution should be used in those with severe renal and hepatic impairment. Teriparatide should also be used with caution in patients with active or recent urolithiasis. No dosage adjustment is required with renal insufficiency, though bioavailability and half-life may increase when $\mathrm{Cl}_{\text {cr }}<30 \mathrm{~mL} /$ minute. One must ensure that there is not a resultant pre-existing hyperparathyroid state due to severe renal insufficiency, though, before proceeding with teriparatide use. Teriparatide is contraindicated in those with hypercalcemia and hyperparathyroidism.

As stated above, the FDA black box warning limiting therapy duration to 2 years relates to the association of teriparatide with an increased risk of osteosarcoma in rat studies. This risk was related to both dose and duration of the medication. However, one limitation of these rat studies is that very high doses were used in the rat's short life cycle. In the original study, the rats were treated for nearly their entire life span (70\% to $80 \%$ of their lifetime) and during the rapid phase of longitudinal skeletal growth (starting at approximately 2 months of age). The rats were also treated with relative supratherapeutic doses for their size. Also, interestingly, the studied Fischer-344 rats have a 1000-fold 
higher baseline risk of osteosarcoma as compared to humans prior to any treatment. ${ }^{25}$

In contrast, the intended clinical use of teriparatide in humans only encompasses $2 \%-3 \%$ of the human life span, and is given to adults, in the setting of a mature skeleton with already closed epiphyseal plates. Subsequently, a repeat study was done by the same researchers looking at osteosarcoma risks as it relates to age at treatment as well as dose range (varying between 3 and 60 times higher than what is approved for human use). They found that when doses more compatible with human use were used, no increased osteosarcoma risk was seen. ${ }^{26}$

Since the launch of the medication in December 2002, Eli Lilly has maintained a safety monitoring program and has identified one possible case of osteosarcoma in a patient treated with Forteo ${ }^{\circledR}$. This was a postmenopausal woman in her seventies with a complex medical history who, into her second year of therapy, was found to have metastatic cancer. She subsequently died, and no autopsy was performed. The primary cancer site was never identified; however, the clinical suspicion was lung cancer with metastases. The pathology consultant felt that the differential diagnosis included several tumor types, including an osteosarcoma variant. In 2006, Eli Lilly submitted the biopsy materials to another bone pathology expert who concluded the lesion was an osteosarcoma. ${ }^{27}$

As of $2006,>250,000$ patients in the US and $>300,000$ patients worldwide were being treated with Forteo ${ }^{\circledR}$. Given that the background incidence of osteosarcoma in the general population of 60-year-old men and women is 1:250,000 per year, it is difficult to assume causality between Forteo ${ }^{\circledR}$ and osteosarcoma. ${ }^{28}$ Between 2002 and 2009, there have been 1 million scripts for Forteo ${ }^{\circledR}$; therefore, just simply based on population prevalence alone, there would have been 4 expected cases of osteosarcoma to date. ${ }^{29}$ However, underreporting always remains a possibility. Eli Lilly is continuing to monitor for osteosarcoma in patients treated with their product. As a result, teriparatide use should be avoided in patients with an increased baseline risk of osteosarcoma, which include those with Paget's disease, prior radiation therapy (external or seed implants), unexplained elevations of alkaline phosphatase, or in young adults with open epiphyses. Similarly, it should not be used in pregnant women.

A theoretical potential exists for teriparatide to accelerate local tumor extension if non-primary bone tumors are unknowingly present in bone, as anabolic therapy increases bone turnover. This explains the hesitancy to use teriparatide in patients with active or recent non-osteosarcoma cancers that have a likelihood to metastasize to bone, such as breast, prostate, lung, thyroid, or kidney. ${ }^{29}$

\section{Side effects}

Teriparatide is generally well tolerated. In clinical trials, the frequency of at least 1 episode of transient hypercalcemia in the 4 to 6 hours after Forteo ${ }^{\circledR}$ administration was $11 \%$ of women and $6 \%$ of men. The number of patients treated with hypercalcemia verified on consecutive measurements was $3 \%$ in women and $1 \%$ in men. Nausea, dizziness, headache, and leg cramps can occur in $7 \%$ to $10 \%$ of patients; however, studies evaluating the $20 \mu \mathrm{g}$ dose reported rates of nausea and headache similar to those who received placebo. ${ }^{9,30,31}$ Rarely rhinitis, or arthralgias can occur, but these are usually not severe enough to require discontinuation. Clinically insignificant decreases in serum magnesium and increases in serum urate levels are seen in both the $20 \mu \mathrm{g}$ and $40 \mu \mathrm{g}$ dosing. Antibodies to teriparatide have been noted in about $3 \%$ of women with long-term treatment; however hypersensitivity reactions or decreased efficacy has not been seen. Post marketing case reports of acute dyspnea, allergic reactions, edema (facial/oral), hypercalcemia $>13 \mathrm{mg} / \mathrm{dL}$, injection site reactions (bruising, pain, swelling), muscle spasm, and urticaria have also been reported. None of these adverse events occurred more frequently than those with placebo or other comparative medications. Side effects did not vary by patient age or those with mild to moderate renal impairment. ${ }^{8}$

\section{Monitoring Before initiation}

It is recommended to obtain a serum calcium, alkaline phosphatase, and serum intact PTH before the initiation of therapy with teriparatide, as baseline elevations may contraindicate therapy. As with all OP treatment regimens, adequate calcium and vitamin D supplementation is essential for efficacy of treatment. Ensuring vitamin D sufficiency (with measurement of serum 25-OH D) before treatment is of utmost importance, due to the widespread prevalence of insufficiency (especially north of Atlanta, Georgia), as well as the possible secondary hyperparathyroid state that can result from low vitamin D levels.

Most postmenopausal women require a daily elemental calcium intake of 1200 to $1500 \mathrm{mg} .{ }^{1}$ This is the total including dietary intake as well as supplement levels. Daily ingestion of foods rich in calcium (ie, dairy, dark green leafy vegetables) are encouraged with supplementation to meet the remaining requirements. Calcium supplementation should 
be divided into $500 \mathrm{mg}$ doses, as doses beyond this exceed the renal threshold for absorption. Calcium carbonate needs an acidic $\mathrm{pH}$ for absorption whereas citrate formulations do not. Thus, women taking concomitant antacids and/or with co-morbidities that alter gut ph may benefit preferentially from calcium citrate supplements.

Many individuals north of the Atlanta region require vitamin D3 supplementation of at least 1000 to 2000 IU D3/day to achieve a mid-range goal of $25 \mathrm{OH}$ vitamin $\mathrm{D}$ of 40 to $50 \mathrm{ng} / \mathrm{mL}$. One can expect a rise in serum of $1 \mathrm{ng} / \mathrm{mL} / 100 \mathrm{IU} /$ day cholecalciferol (D3) and 3 months is needed to establish a new steady state. ${ }^{1}$

\section{During therapy}

Though no specific recommendations for blood test monitoring during therapy is recommended in the package insert, obtaining calcium, phosphorus, and bone-specific alkaline phosphatase levels periodically is recommended.

Serum calcium levels should not be checked any earlier than at least 12 hours after the administration of the medication due to the transient hypercalcemia that can be seen. If hypercalcemia develops, then oral calcium intake should be reduced by $50 \%$, and if calcium elevation persists then all external sources of calcium supplementation should be stopped. Discontinuation of teriparatide is rarely necessary as a result of induced hypercalcemia. ${ }^{29}$

Investigators have begun to evaluate the use of bone formation and turnover markers to follow therapy with multiple bone agents. Increases in bone formation markers (particularly P1NP) are seen within 1 month of treatment and seem to predict gains in BMD during anabolic therapy. Bone resorption markers actually increase as well. It is not yet clear at present, though, that such monitoring can predict fracture efficacy, and thus its clinical use is not well defined at this time. ${ }^{32,33}$ One might consider measuring serum N-terminal P1NP at baseline and following it after 3 months, as this change had a correlation coefficient of 0.65 for predicting the 18-month lumbar spine BMD response. ${ }^{32}$

\section{Post-treatment care}

Although Lindsay et al showed sustained fracture prevention after anabolic therapy cessation, the exact duration of this benefit is uncertain. ${ }^{34}$ In the months and years after treatment with anabolic therapy, a significant amount of the bone density gained during treatment appears to be lost if no further therapy is implemented. Black et al looked at effect on BMD that followed anabolic treatment [rhPTH (1-84)] treatment with placebo vs. continued alendronate treatment in postmenopausal women. Women who had ever been treated with BPS for more than 12 months or for shorter intervals in recent periods prior to the study were excluded. Though the rhPTH (1-84) treatment was only for 1 year, alendronate therapy after rhPTH (1-84) significantly increased BMD in comparison to the results for placebo after PTH, a difference particularly evident for BMD in trabecular bone of the spine on QCT measurement (increase of 31\% in the rhPTH-alendronate group vs $14 \%$ in the rhPTH-placebo group). During year 2, subjects receiving placebo lost substantial BMD. ${ }^{35}$ Therefore, administering alendronate after a course of anabolic therapy appears to conserve the bone gained during that treatment and adds some density on its own. The additional density added is roughly similar in magnitude to the short-term effect of BPS given to previously untreated patients. ${ }^{36}$ Though alendronate is the post-treatment antiresorptive agent commonly evaluated in clinical trials, some sort of antiresorptive should follow the use of teriparatide.

Kaufman et al also showed gradual decline of lumbar spine and total hip BMD with discontinuation of teriparatide after 30 months in men, although the loss was not complete with BMD still remaining higher than baseline. Similarly, these declines were abated with the addition of subsequent antiresorptive therapy post anabolic treatment with resultant further increases in BMP. ${ }^{37}$

Most have utilized treatment with teriparatide according to the package insert, with daily therapy for a maximum of 24 months followed by available alternative therapy thereafter, preferably, BPS. Cosman et al has been studying variations in this usual sequencing for those at highest risk of fracture. Her group recognized that the maximal anabolic window with anabolic agents is within the first 3 to 6 months of therapy based on results of bone marker analysis. They also noted that there is a seeming resistance to teriparatide's effectiveness in time beyond this first 6 months of use, with bone turnover levels subsequently plateauing between 6 to 12 months after teriparatide initiation. They then theorized that a series of intermittent, shorter treatment durations may create multiple anabolic windows that would result in a more significant overall treatment response rather than sustained therapy for 24 months. ${ }^{38,39}$ Initial studies to evaluate the effectiveness of such variations of regimens are underway without definitive randomized controlled trial results to date.

\section{Areas for future research}

While much data has accumulated on teriparatide and its use in OP, many questions remain unanswered. It remains unclear what the optimal duration and sequence of anabolic therapy is, 
whether pulsed and repeated vs continuous therapy is superior, whether certain combinations of therapy are preferred and/or to be avoided, and which PTH formulation may be superior, as other formulations of PTH analogues exist and are in development (PTH 1-81 Preotact ${ }^{\circledR}$ approved in the EU). Additionally, which subsets of patients may best benefit from its use and when also remain in question. Finally, hip fracture reduction has yet to be proven with teriparatide therapy and requires further study, as well as comparative effectiveness trials. Newer agents for OP are on the very near horizon within the US including strontium ranelate, which has partial anabolic and antiresorptive properties. Denosumab, a monoclonal antibody rank ligand inhibitor, if approved, will be the first biologic therapy for bone health. How to utilize such agents in relation to teriparatide is also uncertain.

\section{Disclosures}

The authors declare no conflicts of interest.

\section{References}

1. National Osteoporosis Foundation, Clinician's Guide to Prevention and Treatment of Osteoporosis. Washington, DC: National Osteoporosis Foundation, 2009, 1-36. http://www.nof.org/professionals/ NOF_Clinicians_Guide.pdf. Accessed March 3, 2010.

2. Frolik CA, Black ED, Cain RL, et al. Anabolic and catabolic bone effects of human parathyroid hormone (1-34) are predicted by duration of hormone exposure. Bone. 2003;33:372-379.

3. Tam CS, Heersche JN, Murray TM, et al. Parathyroid hormone stimulates the bone apposition rate independently of its resorptive action: differential effects of intermittent and continuous administration. Endocrinology. 1982;110:506-512.

4. Jiang Y, Zhao JJ, Mitlak BH, et al. Recombinant human parathyroid hormone (1-34) [teriparatide] improves both cortical and cancellous bone structure. J Bone Miner Res. 2003;18:1932-1941.

5. Zanchetta JR, Bogado CE, Ferretti JL, et al. Effects of teriparatide [recombinant human parathyroid hormone (1-34) on cortical bone in postmenopausal women with osteoporosis. J Bone Miner Res. 2003;18:539-543.

6. Dobnig H, Turner RT. Evidence that intermittent treatment with parathyroid hormones increases bone formation in adult rats by activation of bone lining cells. Endocrinology. 1995;136:3632-3638.

7. Qin L, Li X, Ko JK, et al. Parthyroid hormone uses multiple mechanisms to arrest the cell cycle progression of osteoblastic cells from G1 to S phase. J Biol Chem. 2005;280:3104-3111.

8. Blick SKA, Dhillon S, Keam SJ. Teriparatide - a review of its use in osteoporosis. Drugs. 2008;68:2709-2737.

9. Neer RM, Arnaud CD, Zanchetta JR, et al. Effect of parathyroid hormone (1-34) on fractures and bone mineral density in postmenopausal women with osteoporosis. N Engl J Med. 2001;344:1434-1441.

10. Prevrhal S, Krege JH, Chen P, et al. Teriparatide vertebral fracture risk reduction determined by quantitative and qualitative radiographic assessment. Curr Med Res Opin. 2009;25:921-928.

11. Boonen S, Marin F, Obermayer-Pietsch B, et al. Effects of previous antiresorptive therapy on the bone mineral density response to two years of teriparatide treatment in 30 postmenopausal women with osteoporosis. J Clin Endocrinol Metab. 2008;93:852-860.

12. Ettinger B, San Martin J, Crans G, et al. Differential effects of teriparatide on $\mathrm{BMD}$ after treatment with raloxifene or alendronate. $J$ Bone Miner Res. 2004;19:745-751.
13. Eastell R, Nickelsen T, Marin F, et al. Sequential treatment of severe PMOP following teriparatide: final results of the randomized, controlled European study of Forsteo (EUROFORS). J Bone Miner Res. 2009;24: 726-736.

14. Miller PD, Delmas PD, Lindsay R, et al. Early responsiveness of women with OP to teriparatide after therapy with alendronate or risedronate. J Clin Endocrinol Metab. 2008;93:3785-3793.

15. Body JJ, Gaich Ga, Scheele WH, et al. A randomized double blind trial to compare the efficacy of teriparatide 9rh PTH (1-34) with alendronate in postmenopausal women with osteoporosis. J Clin Endocrinol Metab. 2002;87:4528-4535

16. Saag KG, Zanchetta JR, Devogelaer JP, et al. Teriparatide versus alendronate for treatment of glucocorticoid-induced osteoporosis: 36-month results. J Bone Miner Res. 2008;23(Suppl 1):S49.

17. Black DM, Greenspan SL, Ensrud KE, et al. The effects of parathyroid hormone and alendronate alone or in combination in postmenopausal osteoporosis. N Engl J Med. 2003;349:1207-1215

18. Finkelstein JS, Hayes A, Hunzelman JL, et al. The effects of parathyroid hormone, alendronate, or both in men with OP. $N$ Engl J Med. 2003;349;1216-1226.

19. Cosman F, Wermers, RA, Recknor C, et al. Efficacy of adding teriparatide vs switching to teriparatide in PM women with osteoporosis previously treated with raloxifene or alendronate. J Bone Miner Res. 2007;22(Suppl 1):S127.

20. Gasser JA, Ingold P, Venturiere A, Green JR. Chronic subcutaneous, but not single intravenous dosing of rats with bisphosphonates results in reduced bone anabolic response to PTH. J Bone Miner Res. 2006;21(Suppl 1):S113

21. Lindsay R, Nieves J, Formica C, et al. Randomised controlled study of effect of parathyroid hormone on vertebral-bone mass and fracture incidence among postmenopausal women on oestrogen with osteoporosis. Lancet. 1997;350:550-555.

22. Deal C, Omizo M, Schwartz EN, et al. Combination teriparatide and raloxifene therapy for postmenopausal osteoporosis: results from a 6-month double-blind placebo controlled trial. J Bone Miner Res. 2005;20:1905-1911.

23. Lundkvist J, Johnell O, Cooper C, et al. Economic evaluation of parathyroid hormone $(\mathrm{PTH})$ in the treatment of osteoporosis in postmenopausal women. Osteoporos Int. 2006;1:201-211.

24. Center for Drug Evaluation and Research. Clinical pharmacology and biopharmaceutics review online. URL: http:/www.fda.gov/cder/foi/ nda/2002/21-318_FORTEO_BioPharmr.pdf. Accessed Oct 2009.

25. Vahle JL, Sato M, Long GG, et al. Skeletal changes in rats given daily subcutaneous injections of recombinant human parathyroid hormone (1-34) for 2 years and relevance to human safety. Toxicol Pathol. 2002;30:312-321.

26. Vahle JL, Long GG, Sandusky G, et al. Bone neoplasms in F344 rats given teriparatide $[\mathrm{rhPTH}(1-34)]$ are dependent on duration of treatment and dose. Toxicol Pathol. 2004;32:426-438.

27. Harper KD, Krege JH, Marcus R, Mitlak BH. Osteosarcoma and teriparatide? J Bone Miner Res. 2007;22:334.

28. Surveillance Research Program National Cancer Institute SEER*Stat Software, version 6.1.4. www.seer.cancer.gov/seerstat. Accessed March 3, 2010.

29. File E, Deal C. Clinical update on teriparatide. Curr Rheumatol Rep. 2009;11:169-176.

30. Saag KG, Shane E, Boonen S, et al. Teriparatide or alendronate in Glucocorticoid-induced OP. N Engl J Med. 2007;357:2028-2039.

31. Reeve J. Recombinant human parathyroid hormone. BMJ. 2002;324: 435-436.

32. Chen P, Satterwhite JH, Licata AA, et al. Early changes in biochemical markers of bone formation predict BMD response to teriparatide in postmenopausal women with osteoporosis. J Bone Miner Res. 2005;20:962-970.

33. Bauer DC, Garnero P, Bilezikian JP, et al. Short-term changes in bone turnover markers and bone mineral density response to parathyroid hormone in postmenopausal women with osteoporosis. J Clin Endocrinol Metab. 2006;91:1370-1375. 
34. Lindsay R, Zhou H, Cosman F, et al. Effects of a one month treatment with PTH (1-34) on bone formation on cancellous, endocortical, and periostial surfaces of the human ilium. J Bone Miner Res. 2007;22:495-502.

35. Black DM, Bilezikian JP, Ensrud KE. One Year of Alendronate after one year of parathyroid hormone (1-84) for osteoporosis. NEngl J Med. 2005;353:565-565.

36. Heaney RP, Recker RR. Combination and sequential therapy for osteoporosis. N Engl J Med. 2005;353:624-625.
37. Kaufman JM, Orwoll E, Goemaere S, et al. Teriparatide effects on vertebral fractures and bone mineral density in men with osteoporosis: treatment and discontinuation of therapy. Osteoporos Int. 2005;16: 510-516.

38. Cosman F, Nieves J, Zion M, et al. Retreatment with teriparatide one year after the first teriparatide course in patients on continued long-term alendronate. J Bone Miner Res. 2009;24:1110-1115.

39. Cosman F, et al. Current Medical Evidence. 2009;2:1-11.

\section{Publish your work in this journal}

The International Journal of Women's Health is an international, peerreviewed open-access journal publishing original research, reports, reviews and commentaries on all aspects of women's healthcare including gynaecology, obstetrics, and breast cancer. Subject areas include: Chronic conditions (migraine headaches, arthritis, osteoporosis);
Endocrine and autoimmune syndromes; Sexual and reproductive health; Psychological and psychosocial conditions. The manuscript management system is completely online and includes a very quick and fair peer-review system. Visit http://www.dovepress.com/ testimonials.php to read real quotes from published authors. 